\title{
DATA MINING PREDICTIVE MODELS FOR PERVASIVE INTELLIGENT DECISION SUPPORT IN INTENSIVE CARE MEDICINE
}

\author{
Filipe Portela, Filipe Pinto, Manuel Filipe Santos \\ Centro Algoritmi, Universidade do Minho, Portugal \\ cfp@dsi.uminho.pt,fpinto@estg.ipleiria.pt,mfs@dsi.uminho.pt
}

Keywords: Data Mining, KDD, Real-Time, Pervasive, Intelligent Decision Support System, Intensive Care.

\begin{abstract}
The introduction of an Intelligent Decision Support System (IDSS) in a critical area like the Intensive Medicine is a complex and difficult process. In this area, their professionals don't have much time to document the cases, because the patient direct care is always first. With the objective to reduce significantly the manual records and, enabling, at the same time, the possibility of developing an IDSS which can help in the decision making process, all data acquisition process and knowledge discovery in database phases were automated. From the data acquisition to the knowledge discovering, the entire process is autonomous and executed in real-time. On-line induced data mining models were used to predict organ failure and outcome. Preliminary results obtained with a limited population of patients showed that this approach can be applied successfully.
\end{abstract}

\section{INTRODUCTION}

The most important feature for the success of an Intelligent Decision Support System (IDSS) is its capability to operate autonomously and in real-time, providing the results in the right moment of the decision maker need. IDSS operating in critical environments must overcome the difficulty in obtaining the data, processing and transforming them automatically and in real-time.

INTCare is an IDSS for intensive medicine and uses Data Mining (DM) models to predict organ failure and outcome (Gago et al., 2006; Vilas-Boas, Santos, Portela, Silva, \& Rua, 2010).

In order to meet its goals, it has been necessary to change the environment and the information system, developing a real-time data acquisition and processing system (Filipe Portela, Santos, \& VilasBoas, 2012; Manuel Filipe. Santos et al., 2011; M. F. Santos et al., 2009). This system can automatically receive and process the patient data, making it available to obtain further knowledge. Then, it is important to explore the possibility to obtain, clean, validate and transform the data automatically, according to the variables in use by data mining in an adaptive and real way. The intensive care units are known as a place where the patient direct care is in the first place delegating tasks like the health recording to a secondary importance (Lyerla, LeRouge, Cooke, Turpin, \& Wilson, 2010). In the past, we have obtained some good results by using offline data, where it was correctly identified most of the variables in use in this project. In an attempt to transpose this idea to a real environment with real data obtained in a continuous way, it was verified that none of variables were regularly collected in an electronic mode.

In a first step, it was implemented a solution to obtain all the necessary data. Some changes were performed and an Electronic Nursing Record (ENR) has been developed to register and validate the values. Then, it was necessary to develop a data processing system. To automate the entire process, in particular the processing and transformation phases, it was necessary to deploy a set of agents (Manuel Filipe. Santos, et al., 2011; Wooldridge, 1999) which perform some predefined tasks. These agents are responsible to perform all the tasks which in the past were done manually. For example, they are responsible by data acquisition of vital signs, the validation of the collected values, the data mining preparation and the models induction. After all the processes have been totally tested and prepared to be executed automatically without manual efforts, some 
data mining models were developed. The main objective of these DM Models is the use of the obtained results to understand the viability to automate the data transformation process, assessing the quality of the results. These results were compared with the earlier obtained results. All the tasks were carefully implemented and tested using the real data related to the patients admitted in ICU of Hospital Santo António, Centro Hospitalar do Porto in Portugal. This paper will show the procedures implemented for the data transformation process, the first results obtained using DM techniques and finally the analysis of these results.

The work is organized according to the Knowledge Discovering Phases. After this introduction, the second chapter gives an outline of the paper and an overview of the data acquisition system in use. The next four chapters explain the overall KDD phase: at first, it is presented a summary about the first two phases and then all automation of the transformation process is exposed. Fifth chapter introduces the data mining models and the configurations used. The sixth and seventh chapters are related to the evaluation of the results obtained by DM and to the conclusions respectively. In this last chapter, it is also presented the future work.

\section{BACKGROUND}

\subsection{OFFLINE vs. ONLINE}

The former results obtained using EURICUS database (Silva, Cortez, Santos, Gomes, \& Neves, 2008) was the main motivation to develop this work. In this work the variables used were collected manually and in an offline mode: "The data was monitored, collected and registered manually, every hour, all ICU patient biometrics were recorded in a standardized sheet form by the nursing staff. The adverse events were also assigned in a specific sheet at an hourly basis."(Silva, et al., 2008). The variables used were: Age, Critical Events, Admission Variables, Outcome, and SOFA (J. Vincent et al., 1998; J. L. Vincent et al., 1996).

The objective is now to obtain all of those variables automatically and to induce data mining models using an online approach in order to predict the organ failure and outcome in real time. The greater challenge is the development of some procedures which use all values obtained by the data acquisition system instead of using hourly values. This change allows for a continuous data monitoring

\subsection{INTCare System}

INTCare system is composed by four subsystems: Data acquisition, knowledge management, Inference and Interface. Each subsystem is autonomous and uses intelligent agents to perform tasks. Figure 1 presents the current INTCare System and its agents. In this work some of these Agents were used (Manuel Filipe. Santos, et al., 2011). For data acquisition were used the following agents: Gateway, Vital Signs Acquisition agent, ENR agent, LR agent and AIDA. Furthermore, it was used a preprocessing agent for the data validation and transformation. Finally, the induction of data mining models was assured by the Data Mining agent. During the development of INTCare, some features were added according to the pervasive health care concept (Orwat, Graefe, \& Faulwasser, 2008; Varshney, 2007), making the system available anywhere and anytime (Filipe Portela, Santos, Silva, Machado, \& Abelha, 2011)

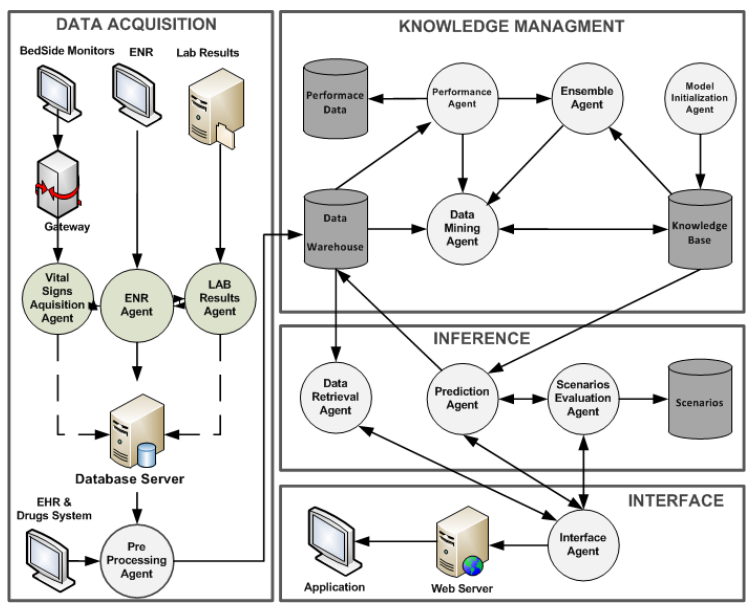

Figure 1: INTCare System

\subsection{Knowledge Discovery Process}

Knowledge Discovery from Databases (KDD) process is recognized as a process which can obtain new knowledge using some data. This process is composed by five stages: Selection, Pre-Processing, Transformation, Data Mining and Interpretation (Fayyad, Piatetsky-Shapiro, \& Smyth, 1996).

Figure 2 shows the KDD process for the ICU data. The database is populated with data from seven major sources. The data are selected from the data warehouse to be processed or transformed, depending of the goal to each variable. After this task, the data are available to be presented by the 
ENR and prepared for the creation of Data Mining Models. Finally, all models are evaluated and the obtained knowledge is presented in the INTCare system.

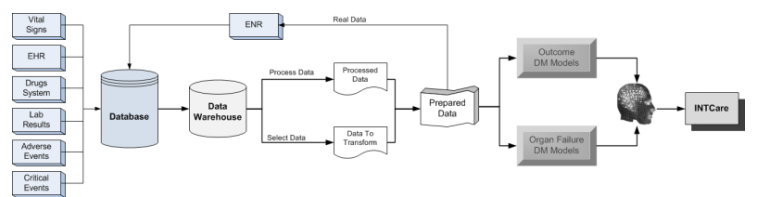

Figure 2: ICU Knowledge Discovery in Database Process

\subsection{Data Mining}

The use of DM techniques in the medical arena has been gaining an increasing interest by researchers but despite the high expectative its application in real world settings has been limited (Bellazzi \& Zupan, 2008). In this case, and considering the targets, it should be treated as a classification problem (Han \& Kamber, 2006). Bearing in mind this point and the idea of having a pervasive and real-time IDSS, a set of DM solutions were explored. However, most of them were discarded due to the fact that they can't receive data from the database, process them and store it again into the database tables in a continuous and quick process. After achieving a set of evaluation and experiments, it was decided to develop prevision models using Oracle Data Mining system (ODM). ODM (Concepts, 2005; Tamayo et al., 2005) was chosen because it gives the possibility to perform all tasks using the database in real-time and are from the same supplier of Hospital database. The techniques of classification used are some of the most used in DM (Wu et al., 2008) such as Support Vector Machine, Decision Trees and Naïve Byes. The data mining results obtained in the past were achieved using other techniques and data with a semiautomatic process and offline method.

\section{DATA SELECTION \& PRE- PROCESSING}

The two initial phases of KDD process (Filipe Portela, et al., 2012) use the data acquisition system presented in the background section (Figure 1) to obtain the data. The first phase is concerned with the data selection from database and is in agreement with what is necessary for the DM Models:

$$
\text { ICU_HL7 } \subseteq\{\text { Vital Signs }\}
$$

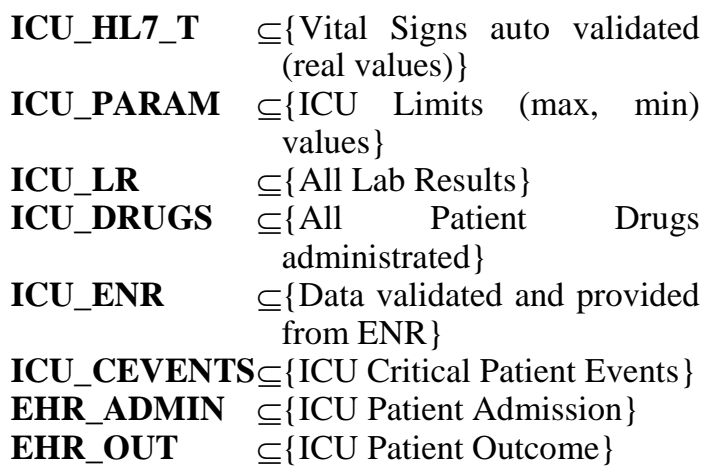

The second phase is responsible for the automatic data validation and patient identification, where it is ensured that all data collected are valid and are correctly identified, i.e. all values collected are between the normal ranges of ICU values, and have a valid patient identification (PID) (F. Portela et al., 2011). At pre-processing phase, other procedures are executed to prepare the Data Mining input table. For example, only the first five days values collected are used. When the patient is admitted to ICU, an agent is responsible to prepare automatically the table adding 120 rows for that patient. When patient goes out, if he/she leaves before 120 hours, the rows in excess are deleted. This table is used as a temporary table of DM input table. In this table are stored: case mix values for all 120 lines and the number of critical events and SOFA $(0,1)$ values for each hour. DM agent gets all variables present in temporary tables and in addition calculates the values in fault.

\section{TRANSFORMATION}

The third phase of KDD process it is autonomous and don't requires a manual action. All tasks were performed automatically and in real-time, by the INTCare intelligent agents. The variables in use are:

SOFA Cardio, Respiratory, Renal, Liver, Coagulation, neurologic $=\{0$, $1\}$;

Case Mix $=\{$ Age (1-4), Admission type $(\mathrm{U}$ or $\mathrm{P})$, Admission from (1-6) $\}$;

Critical Events Accumulated $(\mathrm{ACE})=\{\mathrm{ACE}$ of Blood Pressure $(\mid N)$, ACE of Oxygen Saturation $(\mid \mathrm{N})$, ACE of Heart Rate $(\mid \mathrm{N})$, ACE of Urine Output $(\mid \mathrm{N})\}$

Ratios1 (R1) $=\{$ ACE of BP/elapsed time of stay $(\mathrm{Q}+)$, ACE of SO2/elapsed time of stay $(\mathrm{Q}+), \mathrm{ACE}$ 
of HR/elapsed time of stay (Q+), ACE of Ur/elapsed time of stay $(\mathrm{Q}+)$, Total of ACE / elapsed time of $\operatorname{stay}(\mathrm{Q}+)\}$ \}.

Ratios2 (R2) $=\{$ ACE of BP / max number of ACE of $\mathrm{BP}(\mathrm{Q}+)$, ACE of SO2/ max number of ACE of $\mathrm{SO} 2(\mathrm{Q}+)$, ACE of HR / max number of ACE of HR $(\mathrm{Q}+), \mathrm{ACE}$ of $\mathrm{Ur} / \mathrm{max}$ number of ACE of Ur $(\mathrm{Q}+)$, Total of ACE (Q+), Total of ACE / Total ACE max $(\mathrm{Q}+)\}$.

\section{Ratios $(\mathbf{R})=$ R1 U R2}

ACE of HR: Sum of values hour by hour for each event type. Example if in first hour has 1 event and in the second hour 2, the ACE of second hour is 3 .

Total of ACE is the sum of all ACE of the hour.

Max number of ACE is the number max of each variable present in Table 4.

Elapsed time of stay: Number of hours elapsed since patient admission in the moment of ratio is calculated.

Total ACE max is always $33(10+10+6+7)$ (Table 4).

Outcome $=\{0,1\}$

Table 2 presents the values considered.

Table 1: Variables transforming (example)

\begin{tabular}{|c|c|c|c|c|c|}
\hline & ID & Variable & Min & Max & Value \\
\hline & & - & 18 & 46 & 1 \\
\hline & & - & 47 & 65 & 2 \\
\hline & Age & - & 66 & 75 & 3 \\
\hline & & - & 76 & 130 & 4 \\
\hline & mission & Urgent & - & - & $\mathrm{u}$ \\
\hline & Гуре & Programed & - & - & $\mathrm{p}$ \\
\hline & & Chirurgic & - & - & 1 \\
\hline & & Observation & - & - & 2 \\
\hline & mission & Emergency & - & - & 3 \\
\hline & rom & Other ICU & - & - & 4 \\
\hline & & Other Hospital & - & - & 5 \\
\hline & & Other Situation & - & - & 6 or 7 \\
\hline & & $\mathrm{BP}($ mean) & 0 & 70 & 1 \\
\hline & Cordio & Dopamine & 0,01 & - & 1 \\
\hline & Cardio & Dobutamine & 0,01 & - & 1 \\
\hline $\mathbb{I}$ & & Epi / Norepi & 0,01 & - & 1 \\
\hline O & Renal & Creatinine & 1.2 & - & 1 \\
\hline & Resp & Po2/Fio2 & 0 & 400 & 1 \\
\hline & Hepatic & Bilirubin & 1.2 & - & 1 \\
\hline & Coagul & Platelets & 0 & 150 & 1 \\
\hline
\end{tabular}

\begin{tabular}{|l|l|l|l|l|l|}
\hline & Neuro & Glasgow & 3 & 14 & 1 \\
\hline
\end{tabular}

The first transformation process is a simple task for analysing the values collected and for transforming them according to some rules (if then else). This process is applied to the variables presented in Table 2. When there is a case mix, all variables are inserted in database. When a patient comes into the ICU, a procedure is executed. Regarding to the age parameter, the procedure verifies the patient age. For the admission type and origin, the admission form is consulted in the EHR. In all the cases the values are processed and the value is inserted into DM_INPUT table.

In the case of the SOFA, the approach is a little different. The values are collected in real-time and in a continuous way. The data mining models only use one value per hour. All collected values are considered and the final value is assigned. If more than one result by hour is verified, only the worst value of the hour is considered. For example, in the cardiovascular system, there are five different possibilities (BP, Dopamine, Dobutamine, Epi and Norepi) and it is sufficient if one of them is 1 . The SOFA values are then transformed in binary variables, where 0 describes normality and 1 describes dysfunction/failure and comprises the original SOFA. By default the sofa value variable is 0 and, if some condition is verified (eg. for coagulation, platelets $<=150)$ the values are update to 1 . This update has effect in the starting date which the value was measured. The outcome value (live or died) is updated according to the patient final state. The value in the table is always 0 (live) except if the patient dies. In this case all of the values in the input table are updated to 1 .

The second transformation phase uses critical events. Firstly, a set of procedures are executed in order to understand if a value is critical and if the event is adverse.

Table 2: presents the variables in study and the min and max values for each case.

\begin{tabular}{|c|c|c|c|c|c|c|c|}
\hline EvId & Descr & $\begin{array}{l}\text { Min } \\
\text { EC }\end{array}$ & $\begin{array}{c}\text { Max } \\
\text { EC }\end{array}$ & $\begin{array}{l}\text { Min } \\
\text { Val }\end{array}$ & $\begin{array}{c}\text { Max } \\
\text { Val }\end{array}$ & $\begin{array}{l}\text { Min } \\
\text { Any }\end{array}$ & $\begin{array}{l}\text { Max } \\
\text { Any }\end{array}$ \\
\hline 1011 & $\mathrm{BP}$ & 90 & 180 & 0 & 300 & 60 & \\
\hline 3000 & $\mathrm{O} 2$ & 90 & 100 & 0 & 100 & 80 & \\
\hline 2009 & HR & 60 & 120 & 0 & 300 & 30 & 180 \\
\hline DIU & UR & 30 & 1000 & 0 & 1000 & 10 & \\
\hline
\end{tabular}

At first, it is verified if a value is valid, then it is analysed if that value is critical and how critical is it. According to the Table 3, a value can be considered normal (0), critical (1) or too critical (2). In the case of the value is critical (1), the event will be only 
considered critical if the values collected are verified during some certain time. If the value is spontaneous and is too bad (2), the event will be always critical during the time of the event. This process is presented in the following pseudocode:

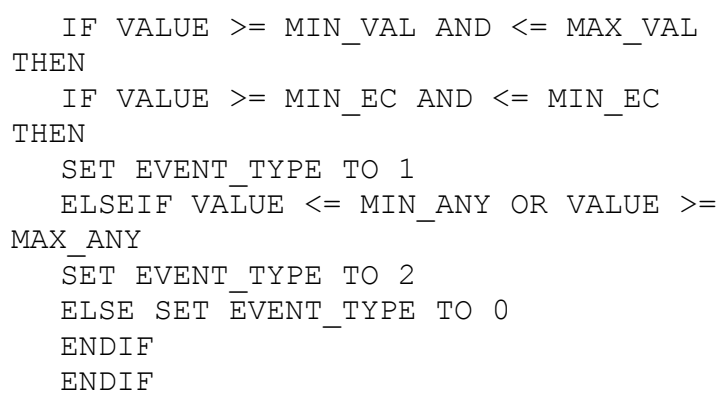

Then each collected value will be inserted in the events table according to the event type and if the predecessor event is or not the same. To know if this event type is of same type a flag will be used.

After know the importance of the values, another procedure starts. This procedure is used to understand if the critical values collected may or may not represent a critical event. To this end, Table 3 is used. To consider an event as a critical event it is necessary to achieve one of the two characteristics. For example, in the case of $\mathrm{SpO} 2$ it is necessary to have values $<=90$ and $>=80$ for more than one hour, or $<=80$ during all the period.

Table 3. The protocol for the out of range physiologic measurements (adapted by Álvaro (Silva, et al., 2008) )

\begin{tabular}{|c|c|c|c|c|}
\hline & $\begin{array}{c}\mathrm{BP} \\
(\mathrm{mmHg})\end{array}$ & $\begin{array}{c}\mathrm{SpO} 2 \\
(\%)\end{array}$ & $\begin{array}{c}\mathrm{HR} \\
(\mathrm{bpm})\end{array}$ & $\begin{array}{c}\mathrm{UR} \\
(\mathrm{ml} / \mathrm{h})\end{array}$ \\
\hline Normal range & $90-180$ & $>=90$ & $60-120$ & $>=30$ \\
\hline Critical event & $>=1 \mathrm{~h}$ & $>=1 \mathrm{~h}$ & $>=1 \mathrm{~h}$ & $>=2 \mathrm{~h}$ \\
\hline Critical event & $<60$ & $<80$ & $\langle 30 \mathrm{~V}\rangle 180$ & $<=10$ \\
\hline
\end{tabular}

After all values are correctly inserted in patient events table, a procedure is executed. This procedure is responsible for reviewing the values according to the event time and type; if a critical event is verified, the event will be inserted in critical events table.

Then, and after have the events are stored in critical events table, an hourly procedure is executed. This procedure calculates the Accumulated Critical Events (ACE) - to reflect the patients' clinical evolution/severity of illness by hour. The next step is the obtaining of the ratios. This process was one of the most difficult to implement due to the need of a real-time calculations in the exact moment when the value is collected. This process requires much memory and processing time and can delay other procedures. For the ratios which use the number of maximum critical events by hour, it is used the maximum number of occurrences verified in the past (Table 4). The max values are updated in the future according to the number max of events verified by a patient for each variable.

Table 4: Critical Events daily number (adapted from (Silva, et al., 2008))

\begin{tabular}{|r|r|r|}
\hline Variables & Min & Max \\
\hline Daily number of critical blood pressure events & 0 & 10 \\
\hline Daily number of critical heart rate events & 0 & 10 \\
\hline Daily number of critical oxygen events & 0 & 6 \\
\hline Daily number of critical urine events & 0 & 7 \\
\hline
\end{tabular}

The next procedure is a selection of the code to calculate the ACE and all ratios to DM model.

During all the processes described above, a procedure is responsible to get all data generated and store them in a specific table for the DM task. Finally, and after having all values correctly inserted in DM input table, another procedure runs to clean the bad values. This procedure is responsible to delete all rows which have null / incorrect values.

\section{REAL-TIME DATA MINING}

After all data have been processed, some models were developed and induced. Only in this step, appears the first manual operation, i.e., the data mining models must be manually configured. 108 models were developed (6 targets x 6 models x 3 techniques). The data mining models were induced in two steps: the first is responsible to prepare the final data to be used by the prediction models; in the second, the data obtained by the first stage are used by $\mathrm{DM}$ techniques to predict the probability of failure of each organ and patient outcome.

In the first step, the data stored in the DM input table is loaded. The numbers (ACE and ratios) are distributed using the Bin Top 7 method. The other values are maintained as they are, and a final table is generated. This table are then used to predict the targets and six models M1 to M6 are induced for each target (renal, hepatic, coagulation, cardiovascular, respiratory and outcome) by each technique (DT, NB, SVM):

$$
\begin{aligned}
& \text { M1 CM + ACE } \\
& \text { M2 CM + ACE + R } \\
& \text { M3 CM + ACE + R1 }
\end{aligned}
$$




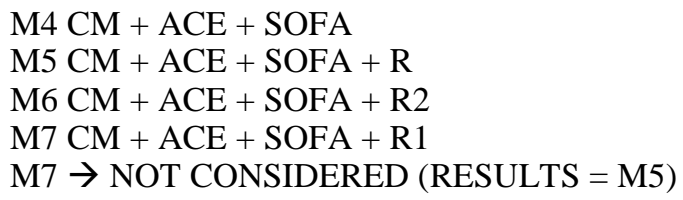

During the modulation of DM, the neurologic system was not considered due to the high number of data in fault.

Figure 3 provides an overview of Data Mining modulation. In this figure it can be observed that the data preparation module is executed using the data stored in database. After the transformation phase, the data are stored in DM_INPUT_DB table. This table is then used to predict the value of six target variables. Afterwards, the obtained results are applied into the prediction table of the patients admitted in ICU (UCI_PATIENT_5DAYS_AG). Then, six new columns are added containing the prediction of happen 1 for each target. In the data mining engine, each target is an individual process.

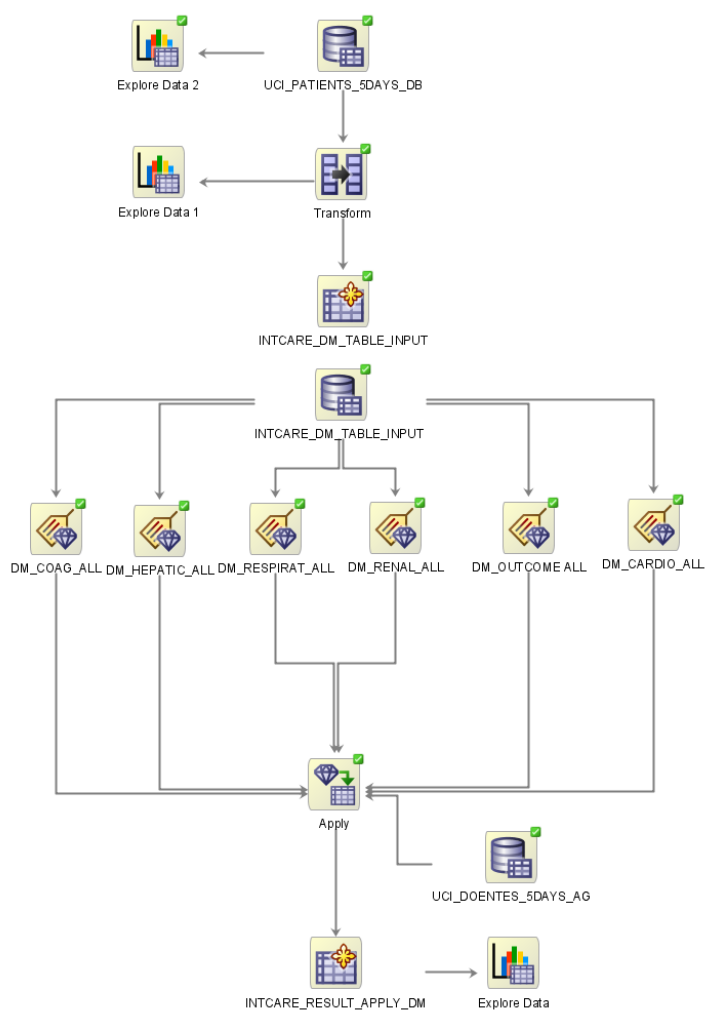

Figure 3: ODM Model

In order to also automate this process, some researche has been done to find how to induce DM models automatically. As result it was possible to develop a procedure to execute the DM engine in real time. The DM agent is responsible to run the engine whenever a request is made. This procedure gets the data stored in UCI_PATIENT_5DAYS_AG table and stores them into a new table (INTCARE_RESULTS_APPLY) combinded with the probability to be 1 for each target.

\section{EVALUATION}

In order to evaluate the models, a test phase using online learning was performed. The Data Mining techniques were applied in the following dataset:

\section{Data Description:}

Collection Time:

Patients Number:

Data Considered:

89 days

109

Exclusion criterion I: Patient with data collecting intermittent, i.e., the collection system failed at least more than one hour in a continuous way;

Exclusion criterion II: Existence of null values;

\section{Data input distribution of the inputs and targets:}

$\begin{array}{lr}\text { NULL RECORDS: } & 0,00 \% \\ \text { AGE = 1: } & 0,00 \% \\ \text { AGE }=2: & 39,64 \% \\ \text { AGE }=3: & 22,85 \% \\ \text { AGE }=4: & 37,51 \% \\ \text { ADMIN FROM = 1: } & 50,45 \% \\ \text { ADMIN FROM = : } & 0,00 \% \\ \text { ADMIN FROM = 3: } & 9,51 \% \\ \text { ADMIN FROM = 4: } & 3,79 \% \\ \text { ADMIN FROM = 5: } & 2,64 \% \\ \text { ADMIN FROM = : } & 21,01 \% \\ \text { ADMIN FROM = 7: } & 40,06 \% \\ \text { ADMIN TYPE = U: } & 59,94 \% \\ \text { ADMIN TYPE = P: } & 40,06 \% \\ \text { OUTCOME = 1: } & 12,58 \% \\ \text { SOFA COAG }=1: & 9,22 \% \\ \text { SOFA HEPATIC = 1: } & 9,85 \% \\ \text { SOFA RENAL = 1: } & 5,16 \% \\ \text { SOFA RESPIRATORY = 1: } & 3,45 \% \\ \text { SOFA CARDIO = 1: } & 27,37 \%\end{array}$

After the DM engine has been run, the best results obtained for each target are presented in Table 5.

Table 5: Results by organ systems and outcome (\%).

\begin{tabular}{|c|c|c|r|r|}
\hline Target & Technique & Scenario & Sensibility & Accuracy \\
\hline Cardio & SVM & M6 & 67.0 & 87.3 \\
\hline Respirat & SVM & M4 & 99.8 & 91.5 \\
\hline
\end{tabular}




\begin{tabular}{|c|c|c|r|r|}
\hline Renal & DT & M5 & 96.4 & 99.3 \\
\hline Liver & SVM & M2 & 100 & 100 \\
\hline Coag & SVM & M6 & 97.5 & 92.6 \\
\hline Out & SVM & M4 & 100 & 99.5 \\
\hline
\end{tabular}

Comparing the results (Table 6) obtained previously (Filipe Portela et al., 2010) and those observed in this study (table 5) it is possible to conclude that some targets (respiratory, liver, coagulation and outcome) present better results. In the opposite direction the cardiovascular target worsened. The models will be used according to the results obtained in terms of sensibility. In a general way, the results are widely satisfactory. Due to the limited dataset (89 patients), we believe that it is possible to improve the results in the future. Duo to the frequency of admissions in the ICU, a more comprehensive study may require at least one year of continuous data acquisition.

Table 6: Variation of the obtained results

\begin{tabular}{|c|r|r|r|r|}
\hline Target & $\begin{array}{l}\text { SENS } \\
\text { OLD }\end{array}$ & $\begin{array}{l}\text { ACC } \\
\text { OLD }\end{array}$ & $\begin{array}{l}\text { SENS } \\
\text { VAR }\end{array}$ & \multicolumn{1}{c|}{$\begin{array}{c}\text { ACC } \\
\text { VAR }\end{array}$} \\
\hline Cardio & 93.4 & 92.7 & -26.4 & -5.4 \\
\hline Respirat & 96.2 & 98.1 & 3.6 & -6.6 \\
\hline Renal & 98.1 & 96.9 & -1.7 & 2.4 \\
\hline Liver & 98.3 & 98.1 & 1.7 & 1.9 \\
\hline Coag & 97.5 & 98.2 & 0 & -5.6 \\
\hline Out & 98.3 & 97.4 & 1.7 & 2.1 \\
\hline
\end{tabular}

\section{CONCLUSIONS}

The process of development of an IDSS which can be autonomous and able to operate in real-time is a big challenge. In this work, the most difficult task has been the transformation of the data process, and the process to obtain critical events and their ratios.

The results proved that it is possible to implement an IDSS in critical health environments minimizing the human intervention.

The greatest contribution of this work is the automation of the entire KDD process, in particular, the data transformation and the data mining processes. INTCare become an autonomous system and is able to, in a automatically and in real-time way, predict the organ failure and outcome for the next 24 hours for the patients admitted in ICU. The DM engine operates autonomously. This engine runs the models and makes the probability results available through the INTCare system.

Experimental work has been conducted in order to compare the sensibility and accuracy values attained using online DM models with those obtained in offline.
In the work has been considered data from a limited number of patients. The obtained results were very good and, in general, similar to the values obtained in the past, using a semi-autonomous process. The doctors can now access to patient data anywhere and anytime, through the electronic nursing record, and they can consult the probability of organ failure and the outcome in an intuitive, quick and easy way. A questionnaire has been prepared in order to understand the level of acceptance of the system. The answers provided by the doctors and by the nurses were very satisfactory and motivating.

Further work will include more data to optimize the DM models. Complementarily, it will be considered the development of an ensemble.

\section{ACKNOWLEDGEMENTS}

The authors would like to thank FCT (Foundation of Science and Technology, Portugal) for the financial support through the contract PTDC/EIA/72819/ 2006. The work of Filipe Portela was supported by the grant SFRH/BD/70156/2010 from FCT.

\section{REFERENCES}

Bellazzi, R., \& Zupan, B. (2008). Predictive data mining in clinical medicine: current issues and guidelines. International journal of medical informatics, 77(2), 81-97.

Concepts, O. D. M. (2005). 11g Release 1 (11.1). Oracle Corp, 2007.

Fayyad, U. M., Piatetsky-Shapiro, G., \& Smyth, P. (1996). From data mining to knowledge discovery: an overview.

Gago, P., Santos, M. F., Silva, Á., Cortez, P., Neves, J., \& Gomes, L. (2006). INTCare: a knowledge discovery based intelligent decision support system for intensive care medicine. Journal of Decision Systems.

Han, J., \& Kamber, M. (2006). Data mining: concepts and techniques: Morgan Kaufmann.

Lyerla, F., LeRouge, C., Cooke, D. A., Turpin, D., \& Wilson, L. (2010). A Nursing Clinical Decision Support System and potential predictors of Head-ofBed position for patients receiving Mechanical Ventilation. American Journal of Critical Care, 19(1), 39-47.

Orwat, C., Graefe, A., \& Faulwasser, T. (2008). Towards pervasive computing in health care - A literature 
review. [10.1186/1472-6947-8-26]. BMC Medical Informatics and Decision Making, 8(1), 26.

Portela, F., Santos, M., Vilas-Boas, M., Rua, F., Silva, Á., \& Neves, J. (2010). Real-time Intelligent decision support in intensive medicine. Paper presented at the KMIS 2010- International Conference on Knowledge Management and Information Sharing.

Portela, F., Santos, M. F., Gago, P., Silva, Á., Rua, F., Abelha, A., et al. (2011). Enabling real-time intelligent decision support in intensive care. Paper presented at the 25th European Simulation and Modelling Conference- ESM'2011, Guimarães, Portugal.

Portela, F., Santos, M. F., Silva, Á., Machado, J., \& Abelha, A. (2011). Enabling a Pervasive approach for Intelligent Decision Support in Critical Health Care. Paper presented at the HCist 2011 - International Workshop on Health and Social Care Information Systems and Technologies.

Portela, F., Santos, M. F., \& Vilas-Boas, M. (2012). A Pervasive Approach to a Real-Time Intelligent Decision Support System in Intensive Medicine. In Springer (Ed.), Communications in Computer and Information Science (Vol. 0272, pp. 14).

Santos, M. F., Portela, F., Vilas-Boas, M., Machado, J., Abelha, A., \& Neves, J. (2011). INTCARE - Multiagent approach for real-time Intelligent Decision Support in Intensive Medicine. Paper presented at the 3rd International Conference on Agents and Artificial Intelligence (ICAART), Rome, Italy.

Santos, M. F., Portela, F., Vilas-Boas, M., Machado, J., Abelha, A., Neves, J., et al. (2009). Information Modeling for Real-Time Decision Support in Intensive Medicine. In S. Y. Chen \& Q. Li (Eds.), Proceedings of the 8th Wseas International Conference on Applied Computer and Applied Computational Science Applied Computer and Applied Computational Science (pp. 360-365). Athens: World Scientific and Engineering Acad and Soc.

Silva, Á., Cortez, P., Santos, M. F., Gomes, L., \& Neves, J. (2008). Rating organ failure via adverse events using data mining in the intensive care unit. Artificial Intelligence in Medicine, 43(3), 179-193.

Tamayo, P., Berger, C., Campos, M., Yarmus, J., Milenova, B., Mozes, A., et al. (2005). Oracle Data Mining. Data Mining and Knowledge Discovery Handbook, 1315-1329.

Varshney, U. (2007). Pervasive healthcare and wireless health monitoring. Mobile Networks and Applications, 12(2-3), 113-127.

Vilas-Boas, M., Santos, M. F., Portela, F., Silva, Á., \& Rua, F. (2010). Hourly prediction of organ failure and outcome in intensive care based on data mining techniques. Paper presented at the 12th International Conference on Enterprise Information Systems.

Vincent, J., Mendonça, A., Cantraine, F., Moreno, R., Takala, J., Suter, P., et al. (1998). Use of the SOFA score to assess the incidence of organ dysfunction/failure in intensive care units : Results of a multicenter, prospective study. Critical care medicine, 26, 1793-1800.

Vincent, J. L., Moreno, R., Takala, J., Willatts, S., De Mendonca, A., Bruining, H., et al. (1996). The SOFA (Sepsis-related Organ Failure Assessment) score to describe organ dysfunction/failure. Intensive care medicine, 22(7), 707-710.

Wooldridge, M. (1999). Intelligent agents Multiagent systems: a modern approach to distributed artificial intelligence (pp. 27-77): MIT Press.

Wu, X., Kumar, V., Ross Quinlan, J., Ghosh, J., Yang, Q., Motoda, H., et al. (2008). Top 10 algorithms in data mining. Knowledge and Information Systems, 14(1), 137. 